\title{
Lower Central Fat Increase Risk of One-Year Muscle Mass Loss in Menopausal Women
}

\author{
Ying-Chou Chen $\mathbb{D}^{1},{ }^{1}$ Wei-Che Lin, ${ }^{2}$ Tien-Tsai Cheng, ${ }^{1}$ Jia-Feng Chen, ${ }^{1}$ Shan-Fu Yu, \\ and Chung-Yuan Hsu' \\ ${ }^{1}$ Department of Rheumatology, Kaohsiung Chang Gung Memorial Hospital, Chang Gung University College of Medicine, \\ Kaohsiung 833, Taiwan \\ ${ }^{2}$ Department of Radiology, Kaohsiung Chang Gung Memorial Hospital, Chang Gung University College of Medicine, \\ Kaohsiung 833, Taiwan
}

Correspondence should be addressed to Ying-Chou Chen; r820713@ms13.hinet.net

Received 17 May 2020; Revised 16 August 2020; Accepted 24 August 2020; Published 2 September 2020

Academic Editor: Sandra Helena Penha Oliveira

Copyright (c) 2020 Ying-Chou Chen et al. This is an open access article distributed under the Creative Commons Attribution License, which permits unrestricted use, distribution, and reproduction in any medium, provided the original work is properly cited.

\begin{abstract}
Background. Hormonal changes had been found in menopausal women. Muscle and bone mass decline after menopause and with aging, increasing the risk for sarcopenia and osteoporosis in later life. Only a few studies suggest that menopausal hormonal changes have an effect on the decline in muscle mass. Objectives. This study aimed at evaluating the risk of muscle mass loss in menopausal women. Materials and Methods. Menopausal women from routine physical health examination were eligible for this study. Muscle mass was determined using dual-energy X-ray absorptiometry at baseline and 1 year later. All of the patients underwent the assessments for liver and kidney function, diabetes, and hypertension, and associated comorbidities were recorded. Results. A total of 172 patients were enrolled. 70 patients had muscle loss at 1 year, and the other 102 did not had loss. The mean age was $70.26 \pm 9.93$ years at the muscle loss group, while $69.25 \pm 10.50$ at the nonprogress group $(p=0.531)$. The mean body mass index was $22.96 \pm 1.91 \mathrm{~kg} / \mathrm{m}^{2}$ at the muscle loss group, while $23.33 \pm 3.71 \mathrm{~kg} / \mathrm{m}^{2}$ at the nonprogress group $(p=0.433)$. The baseline trunk limb fat mass ratio was $1.01 \pm 0.20$ in the muscle loss group and $1.12 \pm 0.26$ in the no muscle loss $(p=0.004)$. Using muscle mass loss as the outcome, logistical regression analysis showed that a baseline trunk limb mass ratio could predict muscle loss, and a higher baseline trunk limb mass ratio was associated with less muscle loss, while a lower trunk limb mass ratio was associated with increased muscle mass loss $(p=0.01)$. Conclusion. This is the first study to investigate the risk of muscle mass loss in menopausal women. Menopausal women with higher central fat had less muscle mass loss, while lower central fat was a risk factor for muscle mass loss. Chronic kidney disease was also a risk factor for muscle mass loss in menopausal women in this study.
\end{abstract}

\section{Introduction}

Hormonal changes such as an increase in serum folliclestimulating hormone (FSH) and decrease in estradiol occur in menopausal women due to ovarian aging and the consequent menopausal transition $[1,2]$. These hormonal changes start 5 years before and continue for years after the final menstrual period [3]. Therefore, studies on the effect of the menopause on women's health and well-being during aging are important. Muscle and bone mass decline after menopause and with aging, increasing the risk of sarcopenia and osteo- porosis in later life. The hormonal changes in menopause are known to lead to bone loss through increased bone turnover with a net deficit in the bone formation relative to bone resorption $[4,5]$. Several studies have also suggested that menopausal hormonal changes have an effect on the decline in muscle mass among middle-aged women $[6,7]$.

The abnormal body composition has been reported in individuals with sarcopenia $[8,9]$, and this has been associated with greater disability and poor long-term outcomes [10] and an increased risk of falls, hip fractures, decreased mobility, and reduced quality of life $[11,12]$. However, few 
studies have examined serial changes in the body composition in menopausal women. Therefore, the aim of this study was to examine changes in the body composition at baseline and after 1 year to identify the factors influencing the body composition changes in menopausal women.

\section{Materials and Methods}

2.1. Study Population. This prospective survey was conducted at the Kaohsiung Chang Gung Memorial Hospital. Menopausal women from routine physical health examination between January 2017 and September 2018 were eligible for this study. Because we intended to examine the body composition changes, we excluded patients who had previously had a stroke and those with systemic infections.

2.2. Outcome Measures. We measured body weight, body mass index, muscle mass, and trunk limb fat mass ratio at baseline and 1 year later. Appendicular muscle mass and trunk limb fat mass ratio were measured using dual energy $\mathrm{X}$-ray absorptiometry. All patients underwent the assessments for liver and kidney function, diabetes, and hypertension, and associated comorbidities were recorded. We defined those with a decrease in the appendicular muscle mass from baseline to 1 year as the muscle loss group, and those with no decrease as the no muscle loss group.

This study was approved by the Ethics Committee of the Kaohsiung Chang Gung Memorial Hospital, and all participants provided written informed consent. The study was conducted according to the tenets of the Declaration of Helsinki.

2.3. Statistical Analysis. Statistical analysis was performed using SPSS software, version 21.0 (SPSS, Chicago, IL, USA). Patient characteristics were reported as simple descriptive statistics (mean $\pm \mathrm{SD}$ ). Comparisons between independent means were analyzed using the $t$-test. Relationships between categorical variables were evaluated using the chi-square test. Logistic regression analysis was used to adjust for the potential confounding factors. A $p$ value $<0.05$ was considered to be statistically significant.

\section{Results}

A total of 172 patients were enrolled, including 70 who had muscle loss at 1 year and 102 who did not. The mean age was $70.26 \pm 9.93$ years in the muscle loss group and 69.25 \pm 10.50 in the no muscle loss group $(p=0.531)$. The mean body mass index was $22.96 \pm 1.91 \mathrm{~kg} / \mathrm{m}^{2}$ in the muscle loss group and $23.33 \pm 3.71 \mathrm{~kg} / \mathrm{m}^{2}$ in the no muscle loss group $(p=0.433)$. The baseline trunk limb fat mass ratio was 1.01 \pm 0.20 in the muscle loss group and $1.12 \pm 0.26$ in the no muscle loss $(p=0.004)$. The muscle loss group had higher rates of diabetes $(p=0.016)$ and chronic kidney disease (CKD) $(p=0.012)$ than the no muscle loss group. The characteristics of the patients are shown in Table 1. Using muscle mass loss as the outcome, logistical regression analysis showed that a baseline trunk limb mass ratio could predict muscle loss, and a higher baseline trunk limb mass ratio was associated with less muscle loss, while a lower trunk limb mass ratio was associated with increased muscle mass loss $(p=0.01)$. Chronic kidney disease was associated with an increase in muscle loss $(p=0.041)$ (Table 2$)$. In subanalysis, we found $6 / 8(75 \%)$ of those with menopause within 5 years had muscle mass loss, while only 64/164 (39\%) had muscle mass loss $(p=0.043)$

\section{Discussion}

Hormonal changes can influence bone and muscle mass in menopause women $[4,5]$, and a change in the body composition has been associated with greater risks of poor healthrelated outcomes and disability [13]. Excess body fat has been associated with a decrease in muscle mass and strength with aging [14], leading to an increased risk of disability [15], institutionalization [16], and mortality [17]. However, these findings are in contrast to ours.

Adipocyte lipolysis has been reported to lead to muscle loss and the leakiness of the respiratory electron-transport chain and also to confer high energy expenditure. Unlike ATP synthase, uncoupling protein 1, which is only expressed in brown adipocytes, has been shown to speed up respiration and convert electrochemical energy into heat production [18]. Moreover, other mitochondria abnormities such as atrophy of oxidative capacity [19] may be involved in muscle weight loss. In a postgastrectomy study, an increase in cytokines was shown to enhance catabolism, leading to a sudden reduction in lean mass. Moreover, body weight was still reduced at least 1 month after surgery due to a reduction in fat mass, which was attributed to a reduced dietary intake [20]. In menopausal woman, dietary factors should also be considered to be an important factor leading to fat loss and muscle loss.

Excess adiposity has been reported to play a role in positive energy balance and decreases in all major components of the total energy expenditure [21] and physical activity [22]. This can then affect the development of sarcopenia, which is aggravated by the increased skeletal muscle fatty infiltration [23], reduced protein intake [24], impaired muscle energetics [25], the increased expression of myostatin [26], altered skeletal muscle substrate metabolism [27], and increased expression of myostatin [26]. However, the exact mechanism in menopausal women is still unclear. In the present study, an increased trunk limb fat mass ratio, meaning central fat, may have influenced the loss of muscle mass. In contrast to decreases in all major components of the total energy expenditure, few studies have reported on how to prevent muscle mass loss in menopausal women. Consequently, a multifaceted approach to the management of menopausal women remains the most promising in terms of reducing the associated health care burden from both a personal and public health perspective.

The key finding of this study is that a higher trunk limb fat mass ratio was associated with a lower risk of muscle mass loss. This may be because adipose fat can protect muscle loss, which has not been reported in other studies. We hypothesize that as female hormones are stored in fat tissue, more fat will result in less muscle loss and less osteoporosis. This is supported by a previous research in which increases in all 
TABLE 1: The characteristics of the study patients.

\begin{tabular}{|c|c|c|c|}
\hline Variables & Muscle mass loss $(n=70)$ & No muscle mass loss $(n=102)$ & $P$ value \\
\hline Age (years), mean \pm SD & $70.26 \pm 9.93$ & $69.25 \pm 10.50$ & 0.531 \\
\hline Body mass index $\left(\mathrm{kg} / \mathrm{m}^{2}\right)$, mean $\pm \mathrm{SD}$ & $22.96 \pm 1.91$ & $23.33 \pm 3.71$ & 0.433 \\
\hline Baseline trunk limb fat mass ratio & $1.01 \pm 0.20$ & $1.12 \pm 0.26$ & 0.004 \\
\hline Smoking $(n, \%)$ & $2(2.9)$ & $2(2.0)$ & 0.538 \\
\hline Alcohol consumption $(n, \%)$ & $2(2.9)$ & $4(3.9)$ & 0.53 \\
\hline Diabetes mellitus $(n, \%)$ & $18(25.7)$ & $12(11.8)$ & 0.016 \\
\hline Hypertension $(n, \%)$ & $22(31.4)$ & $30(29.4)$ & 0.453 \\
\hline Cardiovascular disease $(n, \%)$ & $10(14.3)$ & $10(9.8)$ & 0.253 \\
\hline Chronic liver disease $(n, \%)$ & $6(8.6)$ & $12(11.8)$ & 0.342 \\
\hline Neurological disease $(n, \%)$ & $2(2.9)$ & $6(5.9)$ & 0.296 \\
\hline Chronic kidney disease $(n, \%)$ & $8(11.4)$ & $2(2.0)$ & 0.012 \\
\hline
\end{tabular}

TABLE 2: Factors influencing muscle mass loss.

\begin{tabular}{|c|c|c|c|c|c|}
\hline Variables & Regression coefficient & SE & Wald & $p$ value & OR $(95 \% \mathrm{CI})$ \\
\hline $\begin{array}{l}\text { Trunk/limb } \\
\text { fat mass ratio }\end{array}$ & -3.14 & 0.86 & 13.46 & 0.001 & $0.043 \pm(0.008-0.231)$ \\
\hline Diabetes mellitus & 0.75 & 0.50 & 2.23 & 0.135 & $2.126 \pm(0.790-5.718)$ \\
\hline Chronic kidney disease & 2.12 & 1.03 & 4.19 & 0.041 & $8.300 \pm(1.094-62.985)$ \\
\hline
\end{tabular}

Abbreviations: OR: odds ratio; SE: standard error.

adiposity indices were significantly associated with higher estimated bone mineral density and lower risk of osteoporosis [28]. In addition, another study reported that obesity may have a protective effect, because a higher body weight prevented the loss of muscle mass [29].

We also found that CKD was a risk factor for an increase in muscle mass loss. CKD has been associated with increased frailty and weakness, slow gait, exhaustion, low tolerance for physical activity, and unintentional weight loss [30], and also with muscle wasting, morbidity, and mortality [31]. Worsening the CKD stage and longer dialysis vintage have also been associated with a progressive loss of leg skeletal muscle mass [32]. It is found that chronic kidney disease, and especially dialysis and related lifestyle modification, can greatly impact weight and muscle mass loss. By different types of exercise program, it could be implemented in these patients to improve muscle mass loss [33-35].

The subanalysis of this study showed menopause with 5 years which had more muscle mass progress, so it could explain more about how to prevent the mentioned muscle loss and give directions to further studies that would investigate the interventions to prevent the process of muscle mass loss.

The limitations of this study include the single-center nature, the lack of data on dietary intake and long-term muscle progress, and the small sample size. The strengths of the study include that body composition was analyzed using dual energy X-ray absorptiometry, which is accurate for measuring the human body composition. Further studies are warranted to address the limitations of this study and verify our findings.
In conclusion, this is the first study to investigate the risk of muscle mass loss in menopausal women. Menopausal women with higher central fat had less muscle mass loss, while lower central fat was a risk factor for muscle mass loss. $\mathrm{CKD}$ increased the risk of muscle loss, and this is also an important factor in menopause. Clinicians should monitor muscle mass in menopausal women with lower central fat, because these women are prone to muscle loss.

\section{Data Availability}

All the dataswere availabel as reguested.

\section{Ethical Approval}

This study was approved by the Institutional Review Board of Chang Gung Memorial Hospital. IRB No.: 201601599A3.

\section{Disclosure}

The funding source did not involve in the study design, the collection, the analysis, and the interpretation of data, as well as the writing of the report, and the decision to submit the article.

\section{Conflicts of Interest}

The authors declare that they have no conflicts of interest. 


\section{Authors' Contributions}

Contributors YCC, WCL, and TTC contributed to the conception and design of the work and interpretation of data. They approved the final version. YCC provided the clinical data and samples from patients. JFC and $\mathrm{CYH}$ processed the samples and acquired the data. CYH revised the work critically and approved the final version.

\section{Acknowledgments}

We thank the Kaohsiung Chang Gung Memorial Hospital for the data support. This study was supported by the Chang Gung Memorial Hospital CMRPG8F1731.

\section{References}

[1] S. D. Harlow, M. Gass, J. E. Hall et al., "Executive summary of the stages of reproductive aging workshop +10 : addressing the unfinished agenda of staging reproductive aging," The Journal of Clinical Endocrinology and Metabolism, vol. 97, no. 4, pp. 1159-1168, 2012.

[2] G. Rannevik, K. Carlstrom, S. Jeppsson, B. Bjerre, and L. Svanberg, "A prospective long-term study in women from pre-menopause to post-menopause: changing profiles of gonadotrophins, oestrogens and androgens," Maturitas, vol. 8, no. 4, pp. 297-307, 1986.

[3] G. Rannevik, S. Jeppsson, O. Johnell, B. Bjerre, Y. Laurell-Borulf, and L. Svanberg, "A longitudinal study of the perimenopausal transition: altered profiles of steroid and pituitary hormones, SHBG and bone mineral density," Maturitas, vol. 21, no. 2, pp. 103-113, 1995.

[4] H. Schiessl, H. M. Frost, and W. S. S. Jee, "Estrogen and bonemuscle strength and mass relationships," Bone, vol. 22, no. 1, pp. 1-6, 1998.

[5] H. M. Frost, "On the estrogen-bone relationship and postmenopausal bone loss: a new model," Journal of Bone and Mineral Research, vol. 14, no. 9, pp. 1473-1477, 1999.

[6] G. A. Greendale, B. Sternfeld, M. H. Huang et al., "Changes in body composition and weight during the menopause transition," JCI Insight, vol. 4, no. 5, 2019.

[7] S. C. Ho, S. Wu, S. G. Chan, and A. Sham, "Menopausal transition and changes of body composition: a prospective study in Chinese perimenopausal women," International Journal of Obesity, vol. 34, no. 8, pp. 1265-1274, 2010.

[8] H. Kishimoto, "Change in the definition of osteoporosis especially on bone quality," Clinical Calcium, vol. 15, no. 5, pp. 736-740, 2005.

[9] K. Yamamoto, "Definition and diagnostic criteria of osteoporosis in Japan," Clinical Calcium, vol. 11, no. 1, pp. 19-24, 2001.

[10] H. M. Sosa, “Osteoporosis: the problem of its definition," Medicina Clínica, vol. 124, no. 7, pp. 259-260, 2005.

[11] C. Cooper, W. Dere, W. Evans et al., "Frailty and sarcopenia: definitions and outcome parameters," Osteoporosis International, vol. 23, no. 7, pp. 1839-1848, 2012.

[12] A. A. Sayer, S. M. Robinson, H. P. Patel, T. Shavlakadze, C. Cooper, and M. D. Grounds, "New horizons in the pathogenesis, diagnosis and management of sarcopenia," Age and Ageing, vol. 42, no. 2, pp. 145-150, 2013.
[13] S. Stenholm, D. Alley, S. Bandinelli et al., "The effect of obesity combined with low muscle strength on decline in mobility in older persons: results from the InCHIANTI study," International Journal of Obesity, vol. 33, no. 6, pp. 635-644, 2009.

[14] R. Roubenoff, "Sarcopenic obesity: the confluence of two epidemics," Obesity Research, vol. 12, no. 6, pp. 887-888, 2004.

[15] D. T. Villareal, M. Banks, C. Siener, D. R. Sinacore, and S. Klein, "Physical frailty and body composition in obese elderly men and women," Obesity Research, vol. 12, no. 6, pp. 913-920, 2004.

[16] C. A. Zizza, A. Herring, J. Stevens, and B. M. Popkin, “Obesity affects nursing-care facility admission among whites but not blacks," Obesity Research, vol. 10, no. 8, pp. 816-823, 2002.

[17] T. Rantanen, T. Harris, S. G. Leveille et al., "Muscle strength and body mass index as long-term predictors of mortality in initially healthy men," The journals of gerontology Series A: Biological Sciences and Medical Sciences, vol. 55, no. 3, pp. M168-M173, 2000.

[18] C. Bing, M. Brown, P. King, P. Collins, M. J. Tisdale, and G. Williams, "Increased gene expression of brown fat uncoupling protein (UCP)1 and skeletal muscle UCP2 and UCP3 in MAC16-induced cancer cachexia," Cancer Research, vol. 60, no. 9, pp. 2405-2410, 2000.

[19] C. M. Julienne, J. F. Dumas, C. Goupille et al., "Cancer cachexia is associated with a decrease in skeletal muscle mitochondrial oxidative capacities without alteration of ATP production efficiency," Journal of Cachexia, Sarcopenia and Muscle, vol. 3, no. 4, pp. 265-275, 2012.

[20] T. Aoyama, T. Kawabe, F. Hirohito et al., "Body composition analysis within 1 month after gastrectomy for gastric cancer," Gastric Cancer, vol. 19, no. 2, pp. 645-650, 2016.

[21] D. T. Villareal, C. M. Apovian, R. F. Kushner, S. Klein, American Society for Nutrition, and NAASO, The Obesity Society, "Obesity in older adults: technical review and position statement of the American Society for Nutrition and NAASO, the Obesity Society," Obesity Research, vol. 13, no. 11, pp. 18491863, 2005.

[22] Z. Milanovic, S. Pantelic, N. Trajkovic, G. Sporis, R. Kostic, and N. James, "Age-related decrease in physical activity and functional fitness among elderly men and women," Clinical Interventions in Aging, vol. 8, pp. 549-556, 2013.

[23] R. L. Marcus, O. Addison, L. E. Dibble, K. B. Foreman, G. Morrell, and P. LaStayo, "Intramuscular adipose tissue, sarcopenia, and mobility function in older individuals," Journal of Aging Research, vol. 2012, Article ID 629637, 6 pages, 2012.

[24] S. Sarti, E. Ruggiero, A. Coin et al., "Dietary intake and physical performance in healthy elderly women: a 3-year followup," Experimental Gerontology, vol. 48, no. 2, pp. 250-254, 2013.

[25] M. P. Siegel, T. Wilbur, M. Mathis et al., "Impaired adaptability of in vivo mitochondrial energetics to acute oxidative insult in aged skeletal muscle," Mechanisms of Ageing and Development, vol. 133, no. 9-10, pp. 620-628, 2012.

[26] B. Leger, W. Derave, K. De Bock, P. Hespel, and A. P. Russell, "Human sarcopenia reveals an increase in SOCS-3 and myostatin and a reduced efficiency of Akt phosphorylation," Rejuvenation Research, vol. 11, no. 1, pp. 163-175B, 2008.

[27] D. N. Proctor, W. E. Sinning, J. M. Walro, G. C. Sieck, and P. W. Lemon, "Oxidative capacity of human muscle fiber types: effects of age and training status," Journal of Applied Physiology, vol. 78, no. 6, pp. 2033-2038, 1995. 
[28] H. Tian, J. Pan, D. Qiao et al., “Adiposity reduces the risk of osteoporosis in Chinese rural population: the Henan rural cohort study," BMC Public Health, vol. 20, no. 1, p. 285, 2020.

[29] S. Carbone, C. J. Lavie, and R. Arena, "Obesity and heart failure: focus on the obesity paradox," Mayo Clinic Proceedings, vol. 92, no. 2, pp. 266-279, 2017.

[30] Y. Obi, H. Qader, C. P. Kovesdy, and K. Kalantar-Zadeh, "Latest consensus and update on protein-energy wasting in chronic kidney disease," Current Opinion in Clinical Nutrition and Metabolic Care, vol. 18, no. 3, pp. 254-262, 2015.

[31] S. R. Walker, K. Gill, K. Macdonald et al., "Association of frailty and physical function in patients with non-dialysis CKD: a systematic review," BMC Nephrology, vol. 14, no. 1, p. 228, 2013

[32] S. G. John, M. K. Sigrist, M. W. Taal, and C. W. McIntyre, "Natural history of skeletal muscle mass changes in chronic kidney disease stage 4 and 5 patients: an observational study," PLoS One, vol. 8, no. 5, article e65372, 2013.

[33] Š. Bogataj, M. Pajek, J. Pajek, J. Buturović Ponikvar, and A. H. Paravlic, "Exercise-based interventions in hemodialysis patients: a systematic review with a meta-analysis of randomized controlled trials," Journal of Clinical Medicine, vol. 9, no. 1, p. 43, 2020.

[34] S. Bogataj, J. Pajek, J. Buturovic Ponikvar, V. Hadzic, and M. Pajek, "Kinesiologist-guided functional exercise in addition to intradialytic cycling program in end-stage kidney disease patients: a randomised controlled trial," Scientific Reports, vol. 10, no. 1, article 5717, 2020.

[35] S. Bogataj, M. Pajek, J. Buturovic Ponikvar, and J. Pajek, "Outcome expectations for exercise and decisional balance questionnaires predict adherence and efficacy of exercise programs in dialysis patients," International Journal of Environmental Research and Public Health, vol. 17, no. 9, p. 3175, 2020. 